\title{
Burden of illness in carbapenem-resistant Acinetobacter baumannii infections in US hospitals between 2014 and 2019
}

Jason M. Pogue ${ }^{1}$, Yun Zhou ${ }^{2}$, Hemanth Kanakamedala ${ }^{2}$ and Bin Cai ${ }^{3^{*}(\mathbb{C})}$

\begin{abstract}
Background: Carbapenem-resistant (CR) Acinetobacter baumannii is a concerning pathogen in the USA and worldwide.

Methods: To assess the comparative burden of CR vs carbapenem-susceptible (CS) A. baumannii, this retrospective cohort study analyzed data from adult patients in 250 US hospitals from the Premier HealthCare Database (20142019). The outcomes analyzed included hospital length of stay (LOS), intensive care unit (ICU) utilization, discharge status, in-hospital mortality, readmission rates and hospital charges. Logistic regression was used for univariate and multivariable assessment of the independent relationship between relevant covariates, with a focus on CR status, and in-hospital mortality.

Results: 2047 Patients with CR and 3476 patients with CS A. baumannii infections were included. CR A. baumannii was more commonly isolated in respiratory tract infections (CR 40.7\% and CS 27.0\%, $P<0.01$ ), whereas CS A. baumannii was more frequently associated with bloodstream infections (CS 16.7\% and CR 8.6\%, $P<0.01$ ). Patients with CR A. baumannii infections had higher in-hospital (CR 16.4\% vs CS 10.0\%; $P<0.01$ ) and 30-day (CR 32.2\% vs CS 21.6\%; $P<0.01)$ mortality compared to those with CS infections. After adjusting for age, sex, admission source, infection site, comorbidities, and treatment with in vitro active antibiotics within $72 \mathrm{~h}$, carbapenem resistance was independently associated with increased mortality (adjusted odds ratio 1.42 [95\% confidence interval 1.15; 1.75], $P<0.01$ ). CR infections were also associated with increases in hospital length of stay (CR 11 days vs CS 9 days; $P<0.01$ ), rate of intensive care unit utilization (CR $62.3 \%$ vs CS $45.1 \%$; $P<0.01$ ), rate of readmission with $A$. baumannii infections (CR $17.8 \%$ vs CS $4.0 \% ; P<0.01)$ and hospital charges.
\end{abstract}

Conclusions: These data suggest that the burden of illness is significantly greater for patients with CR A. baumannii infections and are at higher risk of mortality compared with CS infections in US hospitals.

Keywords: Acinetobacter baumannii, Carbapenem resistant, Mortality, ICU utilization, Readmission, Retrospective

\section{Background}

Acinetobacter baumannii has become a concerning nosocomial pathogen that can lead to serious infections including nosocomial pneumonia, bloodstream

*Correspondence: bin.cai@shionogi.com

${ }^{3}$ Shionogi Inc., 300 Campus Drive, Suite 100, Florham Park, NJ 07932, USA

Full list of author information is available at the end of the article infection, skin and skin structure infection, and urinary tract infection $[1,2]$. A. baumannii is an opportunistic, nonfermenting Gram-negative rod and its capability for long-term survival and antimicrobial resistance increases the risk of colonization and infection, particularly in the hospital setting $[2,3]$.

A small retrospective matched cohort study conducted in the USA in the early 2000s demonstrated that the 
presence of multidrug-resistant (MDR) A. baumannii infections in hospitalized patients was associated with increased length of stay in both the hospital and intensive care unit (ICU) compared with susceptible A. baumannii infections or noninfected hospitalized patients [4]. However, this study failed to demonstrate a significant impact on mortality with MDR A. baumannii versus non-MDR A. baumannii [4]. With the increased use of carbapenems, a large proportion of $A$. baumannii have become resistant to carbapenems in the USA and globally [1, 57]. Carbapenem-resistant (CR) A. baumannii remains an urgent threat in the USA according to the 2019 Centers for Disease Control and Prevention (CDC) antimicrobial resistance status report [8]. Previous studies have shown that CR A. baumannii may be associated with poor outcomes [9-12]. The mortality rates in publications over the past two decades for patients with CR, MDR, or extensively drug-resistant $A$. baumannii infections have ranged between 24 and $83 \%$ globally, and patients with multiple comorbidities have a particularly high risk of mortality due to CR A. baumannii infection [13, 14]. Despite these findings, contemporary data assessing the comparative burden of illness and impact on outcomes of infections due to $C R$ versus carbapenem-susceptible (CS) infections remain limited.
The objective of the current retrospective study was to evaluate the burden of illness with regard to mortality, hospitalization, ICU utilization, discharge status, and readmission in patients infected with CR versus CS $A$. baumannii based on a large US hospital-based healthcare database over a five-year period between 2014 and 2019.

\section{Methods \\ Study design and eligibility}

This was a retrospective cohort analysis of anonymized patient data from 250 US hospitals with microbiological data for A. baumannii in the Premier Healthcare Database between 1st January 2014 and 30th June 2019.

Hospitalized adult patients were eligible for inclusion in the current analysis if they had isolation of A. baumannii at various infection sites during the study period, had microbiological susceptibility testing to the carbapenems performed, and received Gram-negative antibiotic treatment within the period of -2 and +3 days of the index culture to minimize the inclusion of potential colonizers (Fig. 1).

Patients were excluded from the analysis if they did not have $A$. baumannii isolated from an index culture, if they were treated in the outpatient setting, were less than 18 years of age, had cystic fibrosis, or did not have

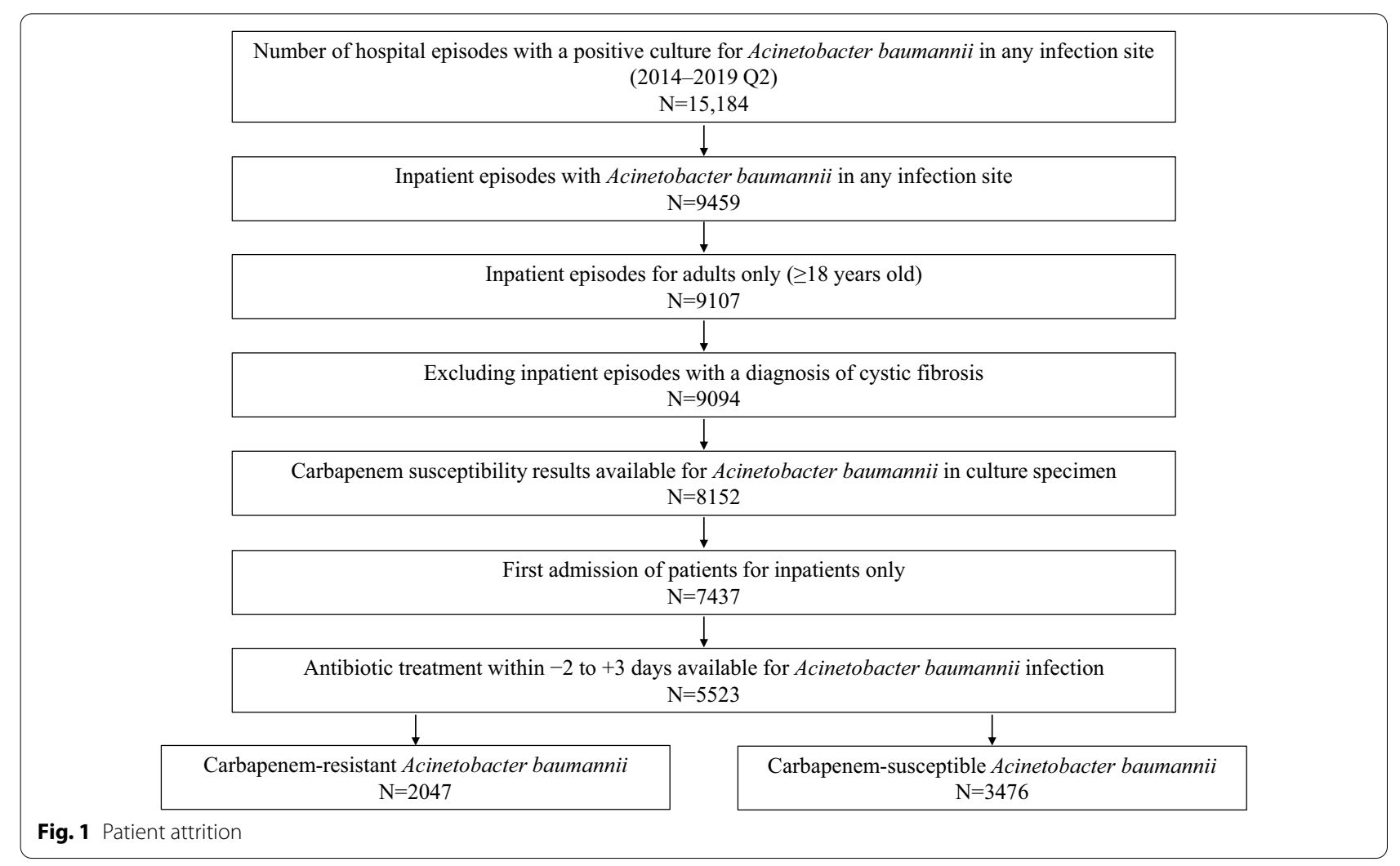


a carbapenem susceptibility test or the susceptibility test results were noninterpretable (Fig. 1).

Following Clinical and Laboratory Standards Institute breakpoints [15], the susceptibility to doripenem, imipenem, or meropenem was determined from the patient's medical chart in order to stratify patients by carbapenem susceptibility status. A. baumannii was defined as carbapenem resistant (CR) if it was resistant or displayed intermediate resistance to any of these three carbapenems. $A$. baumannii was defined as carbapenem susceptible (CS) if it was susceptible to all carbapenems tested (excluding ertapenem). Because patients could have had multiple infections during the study period, the index culture and respective study group were based on the presence or absence of a CR A. baumannii infection occurring at any time within the study period. If patients had any CR $A$. baumannii infection during the study period, they were included in the CR cohort and the index culture was the first culture positive for CR A. baumannii. If patients only had CS A. baumannii isolated, they were included in the CS group and the index culture was the first culture positive for CS A. baumannii during the study period. The index hospitalization was defined as the hospitalization associated with the index culture. Each patient was only included once in the analysis.

The infection site was categorized as blood (bloodstream infection), respiratory (respiratory tract infection), urine (urinary tract infection), wound, or other. If A. baumannii was isolated from multiple specimens from the same patient during the index hospitalization, site of infection was defined as the site from which the first positive culture was obtained on the index culture day.

\section{Outcomes}

The following baseline characteristics were analyzed: demographics (age [years], sex, and race), admission source (nonhealthcare or healthcare facilities), comorbidities, and site of A. baumannii infection (blood, respiratory, urine, wound or other). The main outcome parameters included all-cause in-hospital mortality, overall hospital length of stay (LOS) defined as the number of days from admission to discharge, and infection-associated LOS defined as the number of days from index culture date to discharge.

Other parameters included ICU admission during index hospitalization, ICU-associated LOS, infectionassociated ICU utilization, infection-associated ICULOS, and discharge status (i.e. death, discharged to home, transferred to another institution or hospice, and other). We also assessed hospital charges related to the total duration of hospitalization, the duration of ICU stay, and infection-associated ICU stay, when applicable.
Kaplan-Meier analysis for survival probability by CR status was also performed. The rates of readmission due to any cause, with any Gram-negative organism isolated, or with repeat $A$. baumannii isolated during the readmission were analyzed for patients discharged alive from the index hospitalization.

\section{Statistical analysis}

Descriptive statistics were assessed between patient groups, including $\mathrm{n}$ (\%) for categorical variables and mean (standard deviation $[\mathrm{SD}]$ ) and median (interquartile range [Q1-Q3]) for continuous variables. Univariate comparisons related to baseline characteristics and outcomes between CR and CS patients were conducted using $X^{2}$ test for categorical variables, a Student's $t$ test for mean values, or a Wilcoxon rank sum test for median values for continuous variables. A Kaplan-Meier analysis was conducted to analyze the days between index culture date and death date during the index hospitalization. Log-rank $P$ value of $<0.05$ was used for determining the statistically significant difference between CR and CS group in the probability of death at any time point.

In order to determine the independent impact of carbapenem-resistance on in-hospital mortality, crude and adjusted Odds Ratio (OR) and their 95\% confidence intervals based on normal approximation (Wald) method were calculated using univariate and multivariable logistic regression. Variables included in the multivariable logistic regression were selected based on $P$ value $<0.1$ in the bivariate analysis of each variable and CR status.

The patient information was anonymized and deidentified prior to analysis. Because this study used already existing fully de-identified data, it was exempt from ethics review under US 45 CFR 46.104(d)(4) [16].

\section{Results}

\section{Baseline characteristics}

Of 15,184 screened hospital episodes with a positive culture for $A$. baumannii from any infection site during the study period, a total of 5523 patients were included in the analysis (Fig. 1). Of these, 2047 (37.1\%) patients had CR and 3476 (62.9\%) had CS A. baumannii (Table 1). Patients in the CR A. baumannii cohort were older (median 63 years vs 60 years; $P<0.01$ ) and less likely to be male (58.9\% vs $62.4 \% ; P=0.01$ ) when compared with the CS A. baumannii cohort, respectively (Table 1 ).

The median Charlson Comorbidity Index score of patients with $\mathrm{CR}$ and $\mathrm{CS}$ infections were equivalent (3 vs 3), although higher scores were more frequent in patients with CR infections (Table 1). In line with this, patients with CR infections were more likely to have any comorbid condition $(92.9 \%$ vs $85.6 \% ; P<0.01)$ and had higher frequencies of various comorbidities, including 
Table 1 Baseline characteristics of patients with Acinetobacter baumannii infections, stratified by carbapenem susceptibility status

\begin{tabular}{|c|c|c|c|c|}
\hline Characteristics & $\begin{array}{l}\text { Overall } \\
N=5523\end{array}$ & $\begin{array}{l}\text { Carbapenem resistant } \\
N=2047\end{array}$ & $\begin{array}{l}\text { Carbapenem } \\
\text { susceptible } \\
N=3476\end{array}$ & $P$ value \\
\hline Age, years, median (Q1-Q3) & $61(50.0-72.0)$ & $63(54.0-72.0)$ & $60(48.0-71.0)$ & $<0.01$ \\
\hline Sex, n (\%) & & & & 0.01 \\
\hline Female & 2149 (38.9) & $841(41.1)$ & $1308(37.6)$ & \\
\hline Male & $3374(61.1)$ & $1206(58.9)$ & $2168(62.4)$ & \\
\hline Race, n (\%) & & & & $<0.01$ \\
\hline White & $3648(66.1)$ & $1358(66.3)$ & $2290(65.9)$ & \\
\hline Black & $1155(20.9)$ & $500(24.4)$ & $655(18.8)$ & \\
\hline Other & $641(11.6)$ & $151(7.4)$ & $490(14.1)$ & \\
\hline Unable to determine & $79(1.4)$ & $38(1.9)$ & $41(1.2)$ & \\
\hline Site of index culture infection, $n(\%)$ & & & & $<0.01$ \\
\hline Blood & $756(13.7)$ & $176(8.6)$ & $580(16.7)$ & \\
\hline Respiratory & $1770(32.1)$ & $833(40.7)$ & $937(27.0)$ & \\
\hline Urine & $681(12.3)$ & $233(11.4)$ & $448(12.9)$ & \\
\hline Wound $^{\mathrm{a}}$ & $1970(35.7)$ & $693(33.9)$ & $1277(36.7)$ & \\
\hline Other $^{b}$ & $346(6.3)$ & $112(5.5)$ & $234(6.7)$ & \\
\hline Admission source, n (\%) & & & & $<0.01$ \\
\hline Nonhealthcare facility & $3936(71.3)$ & $1230(60.1)$ & $2706(77.9)$ & \\
\hline Transfer from other facility/hospital & $1137(20.6)$ & $560(27.4)$ & $577(16.6)$ & \\
\hline Transfer from SNF/ intermediate care facility & $345(6.3)$ & $237(11.6)$ & $108(3.1)$ & \\
\hline Other/unavailable & $105(1.9)$ & $20(1.0)$ & $85(2.5)$ & \\
\hline Days between admission and index culture, n (\%) & & & & $<0.01$ \\
\hline 1 Day prior to admission & $217(3.9)$ & $57(2.8)$ & $160(4.6)$ & \\
\hline Same day as admission & $2500(45.3)$ & $734(35.9)$ & $1766(50.8)$ & \\
\hline Day 2 & $907(16.4)$ & $407(19.9)$ & $500(14.4)$ & \\
\hline Day 3 & $352(6.4)$ & $165(8.1)$ & $187(5.4)$ & \\
\hline Day 4 & $208(3.8)$ & 79 (3.9) & $129(3.7)$ & \\
\hline Day 5 & $178(3.2)$ & $93(4.5)$ & $85(2.5)$ & \\
\hline$\geq 6$ days & $1161(21.0)$ & $512(25.0)$ & $649(18.7)$ & \\
\hline \multicolumn{5}{|l|}{ Baseline CCI Score } \\
\hline Median (Q1-Q3) & $3(2.0-5.0)$ & $3(2.0-5.0)$ & $3(1.0-5.0)$ & $<0.01$ \\
\hline $0, \mathrm{n}(\%)$ & $647(11.7)$ & $145(7.1)$ & $502(14.4)$ & $<0.01$ \\
\hline $1, \mathrm{n}(\%)$ & $730(13.2)$ & $227(11.1)$ & $503(14.5)$ & \\
\hline $2, \mathrm{n}(\%)$ & $939(17.0)$ & $383(18.7)$ & $556(16.0)$ & \\
\hline $3-5, \mathrm{n}(\%)$ & $2115(38.3)$ & $843(41.2)$ & $1272(36.6)$ & \\
\hline $5+, n(\%)$ & $1092(19.8)$ & $449(21.9)$ & $643(18.5)$ & \\
\hline \multicolumn{5}{|l|}{ Baseline comorbidities, n (\%) } \\
\hline At least 1 comorbidity & $4876(88.3)$ & $1902(92.9)$ & $2974(85.6)$ & $<0.01$ \\
\hline Myocardial infarction & $681(12.3)$ & $266(13.0)$ & 415 (11.9) & 0.25 \\
\hline Congestive heart failure & $1676(30.4)$ & $724(35.4)$ & $952(27.4)$ & $<0.01$ \\
\hline Peripheral vascular disease & $856(15.5)$ & $332(16.2)$ & $524(15.1)$ & 0.26 \\
\hline Cerebrovascular disease & $584(10.6)$ & $262(12.8)$ & $322(9.3)$ & $<0.01$ \\
\hline Dementia & $345(6.3)$ & $177(8.7)$ & $168(4.8)$ & $<0.01$ \\
\hline Chronic pulmonary disease & $1798(32.6)$ & $780(38.1)$ & $1018(29.3)$ & $<0.01$ \\
\hline Peptic ulcer disease & $102(1.9)$ & $43(2.1)$ & $59(1.7)$ & 0.28 \\
\hline Liver disease & $514(9.3)$ & $157(7.7)$ & $357(10.3)$ & $<0.01$ \\
\hline Diabetes & $2558(46.3)$ & $1083(52.9)$ & $1475(42.4)$ & $<0.01$ \\
\hline Hemiplegia or paraplegia & $826(15.0)$ & 442 (21.6) & $384(11.1)$ & $<0.01$ \\
\hline Renal disease & $1849(33.5)$ & $772(37.7)$ & $1077(31.0)$ & $<0.01$ \\
\hline
\end{tabular}


Table 1 (continued)

\begin{tabular}{|c|c|c|c|c|}
\hline Characteristics & $\begin{array}{l}\text { Overall } \\
N=5523\end{array}$ & $\begin{array}{l}\text { Carbapenem resistant } \\
N=2047\end{array}$ & $\begin{array}{l}\text { Carbapenem } \\
\text { susceptible } \\
N=3476\end{array}$ & $P$ value \\
\hline Any malignancy & $439(8.0)$ & $113(5.5)$ & $326(9.4)$ & $<0.01$ \\
\hline Metastatic solid tumor & $178(3.2)$ & $41(2.0)$ & $137(3.9)$ & $<0.01$ \\
\hline Active drug within $72 \mathrm{~h}$ on or after index culture date ${ }^{c}$ & $2445(44.3)$ & $312(15.2)$ & $2133(61.4)$ & $<0.01$ \\
\hline \multicolumn{5}{|l|}{$\overline{C C I}$ Charlson Comorbidity Index, SNF Skilled nursing facility } \\
\hline \multicolumn{5}{|l|}{ a Wound, bone, body parts, skin, tissue, abscess, ulcer } \\
\hline${ }^{\mathrm{b}}$ Abdomen, genital, sinus, other & & & & \\
\hline
\end{tabular}

diabetes $(52.9 \%$ vs $42.4 \%, P<0.01)$, chronic pulmonary disease (38.1\% vs $29.3 \%, P<0.01$ ), renal disease $(37.7 \%$ vs $31.0 \%, P<0.01)$, congestive heart failure $(35.4 \%$ vs $27.4 \%$, $P<0.01)$, and hemiplegia or paraplegia $(21.6 \%$ vs $11.1 \%$, $P<0.01)$ than patients with CS $A$. baumannii infections (Table 1).

Overall $A$. baumannii was most frequently isolated from wounds (35.7\%), followed by the respiratory tract (32.1\%), blood (13.7\%), urine (12.3\%), or other sources (6.3\%) (Table 1; Additional file 1: Fig. S1). The distribution of CR and CS A. baumannii differed significantly $(P<0.01)$ by site. Respiratory tract infections were more common with $\mathrm{CR}$ isolates $(40.7 \%$ vs $27.0 \%, P<0.01)$, while CS isolates were more common in bloodstream infections (CR $8.6 \%$ vs CS $16.7 \%, P<0.01$ ). CR and CS A. baumannii had been found in similar proportions in urine (CR $11.4 \%$ and CS $12.9 \%, P=0.10$ ) and wounds (CR $33.9 \%$ and CS 36.7\%, $P=0.03$ ) (Table 1; Additional file 1: Fig. S1).

Patients with CR infections were more likely to be transferred from another hospital/facility $(27.4 \%$ vs $16.6 \%, P<0.01)$, or skilled-nursing or intermediate care facility $(11.6 \%$ vs $3.1 \%, P<0.01)$. Approximately half of CS A. baumannii were detected in the index culture on the day prior to or same day of hospital admission (55.4\%), while this rate was $38.6 \%$ for CR $A$. baumannii $(P<0.01)$. $\mathrm{CR}$ patients were more likely to have a nosocomial origin ( $>3$ days after hospital admission) of their pathogen (33.4\% vs $24.9 \%$; $P<0.01$ ) (Table 1 ).

\section{Length of stay and ICU utilization}

In CR $A$. baumannii infections, median LOS was 2 days longer (11 days vs 9 days; $P<0.01$ ) and median infection-associated LOS was 1 day longer (9 days vs 8 days; $P<0.01$ ) than in CS A. baumannii infections (Table 2). Patients with CR $A$. baumannii infections were more often admitted to the ICU for any reason $(62.3 \%$ vs $45.1 \%$; $P<0.01$ ), and to have an index culture during the ICU stay $(45.3 \%$ vs $33.3 \%$; $P<0.01)$ than patients with CS $A$. baumannii infections (Table 2). There was no difference in median ICU LOS (CR 6 vs CS 6, $P=0.98$ ) or infectionassociated ICU LOS (CR 5 vs CS $5, P=0.86$ ) between $\mathrm{CR}$ and CS A. baumannii infections. The overall LOS charges and infection-associated LOS charges were significantly higher for CR infections than for CS infections (Additional file 2: Table S1). ICU-LOS charges and infection-associated ICU-LOS charges were similar between CR and CS A. baumannii infections (Additional file 2: Table S1).

\section{Mortality and discharge status}

Patients with CR A. baumannii infections had higher inhospital mortality $(16.4 \%$ vs $10.0 \% ; P<0.01)$. There was also an increased mortality in patients with $C R$ infections at Day 30 (32.2\% vs 21.6\%; $P<0.01)$ and Day 60 (49.8\% vs $31.2 \% ; P<0.01$ ). Based on the Kaplan-Meier analysis, patients with CR $A$. baumannii infections had a significantly higher probability of dying than those with CS A. baumannii infections (log-rank $P<0.0001$ ) (Fig. 2). Patients with CR $A$. baumannii infections were significantly less likely to be discharged home (16.2\% vs $47.8 \%)$ and significantly more likely to be discharged/transferred to another facility (61.9\% vs $36.1 \%)$ than patients with CS A. baumannii infections $(P<0.01$, Table 2$)$. Patients who were admitted to hospital from a nonhealthcare facility point of origin were less likely to be discharged home if they had CR infections (19.9\%) than if they had CS infections $(51.3 \%)(P<0.01)$.

Among patients with CR A. baumannii infections, the highest mortality rate was observed for bloodstream infections (40.9\%; Table 3) followed by respiratory tract infections $(21.9 \%)$, while mortality was $9.3 \%$ for patients with urinary tract infections and $6.6 \%$ for those with wound infections (Table 3 ). For patients with CS A. baumannii respiratory tract infections, a mortality rate of $23.2 \%$ was found, but the rate was only $12.1 \%$ for patients with bloodstream infections (Table 3). Higher mortality rates for CR than CS A. baumannii infections 
Table 2 Outcomes in patients with Acinetobacter baumannii infections, stratified by carbapenem susceptibility status

\begin{tabular}{|c|c|c|c|c|}
\hline Outcomes & $\begin{array}{l}\text { Overall } \\
N=5523\end{array}$ & $\begin{array}{l}\text { Carbapenem resistant } \\
N=2047\end{array}$ & $\begin{array}{l}\text { Carbapenem } \\
\text { susceptible } \\
N=3476\end{array}$ & $P$ value \\
\hline \multicolumn{5}{|l|}{ Overall LOS ${ }^{\mathrm{a}}$} \\
\hline Overall LOS, days, median (Q1-Q3) & $10(6.0-18.0)$ & $11(7.0-19.0)$ & $9(6.0-17.0)$ & $<0.01$ \\
\hline \multicolumn{5}{|l|}{ Infection-associated LOS ${ }^{b}$} \\
\hline Infection-associated LOS, days, median (Q1-Q3) & $8(5.0-14.0)$ & $9(5.0-14.0)$ & $8(5.0-13.0)$ & $<0.01$ \\
\hline \multicolumn{5}{|l|}{ ICU utilization } \\
\hline ICU admission during hospitalization, n (\%) & $2845(51.5)$ & $1276(62.3)$ & $1569(45.1)$ & $<0.01$ \\
\hline Index culture during ICU stay, n (\%) & $2084(37.7)$ & $927(45.3)$ & $1157(33.3)$ & $<0.01$ \\
\hline ICU-LOS, days, median (Q1-Q3) & $6(2.0-15.0)$ & $6(3.0-14.0)$ & $6(2.0-15.0)$ & 0.98 \\
\hline \multicolumn{5}{|l|}{ Infection-associated ICU utilization } \\
\hline Index culture during infection-associated ICU stay, n (\%) & $2084(37.7)$ & $927(45.3)$ & $1157(33.3)$ & $<0.01$ \\
\hline Infection-associated ICU-LOS, days, median (Q1-Q3) ${ }^{d}$ & $5(2.0-10.0)$ & $5(2.0-10.0)$ & $5(2.0-10.0)$ & 0.86 \\
\hline Discharge status, n (\%) & & & & $<0.01$ \\
\hline Death & $683(12.4)$ & $336(16.4)$ & $347(10.0)$ & \\
\hline Home & $1992(36.1)$ & $332(16.2)$ & $1660(47.8)$ & \\
\hline Hospice & $236(4.3)$ & $98(4.8)$ & $138(4.0)$ & \\
\hline Other & $92(1.7)$ & $14(0.7)$ & $78(2.2)$ & \\
\hline Transfer to other facility & $2520(45.6)$ & $1267(61.9)$ & $1253(36.1)$ & \\
\hline
\end{tabular}

ICU intensive care unit, LOS length of stay

${ }^{a}$ Defined as the number of days from admission to discharge date, regardless of discharge status

${ }^{b}$ Defined as the number of days from index culture date to discharge, regardless of discharge status

'Defined as the number of days from first to the last ICU service day, regardless of discharge status

${ }^{d}$ Defined as the number of days in ICU that was associated with the infection (i.e., index culture was taken within 2 days before ICU admission or during the ICU stay) and calculated as the following: (1) if a positive culture was obtained within 2 days of ICU admission, the entire ICU. LOS was used; (2) if the culture was obtained during ICU stay, the LOS was taken as the number of days from the ICU index culture to discharge from the ICU

was observed in bloodstream, urinary tract, wound, and other types of infections, while mortality rates were similar in patients with respiratory tract infections. Overall, patients were at higher risk of mortality if they were admitted to the ICU during the index hospitalization (CR infections: $23.3 \%$ with ICU vs $5.1 \%$ without ICU; CS infections: $18.9 \%$ with ICU vs $2.6 \%$ without ICU) (Additional file 3: Table S2). The difference in mortality rates between CR and CS infections based on ICU status was consistent across infection sites (Additional file 3: Table S2).

Table 4 displays the association between covariates identified using bivariate analysis (Additional file 3: Table S3) and in-hospital mortality in unadjusted and adjusted regression models. Presence of CR was associated with an increased risk of death in the unadjusted analysis (unadjusted OR: 1.77; 95\% confidence interval: $1.51 ; 2.08 ; P<0.01)$. After controlling for age, race, comorbidities, site of infection, admission source, and receipt of antibiotics with in vitro activity within $72 \mathrm{~h}$ of index culture, CR A. baumannii was independently associated with an increased risk of in-hospital mortality (adjusted OR: $1.42[1.15 ; 1.75], P<0.01)$.

\section{Readmission}

There was no difference in rates of overall readmission due to any cause between patients in the CR and CS $A$. baumannii cohorts (CR 58.4\% vs CS 56.8\%) who were alive at discharge. Compared with patients with CS $A$. baumannii infections, the readmission with any Gramnegative organism isolated (CR $36.4 \%$ vs CS $22.2 \%$; $P<0.01$ ) and with $A$. baumannii isolated (CR $17.8 \%$ vs CS $4.0 \% ; P<0.01$ ) were markedly higher for patients with $C R$ infections (Additional file 5: Table S4, Additional file 6: Table S5). The difference in readmission rates between patients in the CR and CS A. baumannii cohorts was consistent across all infection sites (Additional file 6: Table S5).

\section{Discussion}

The current analysis provides information on the burden of illness due to CR versus CS $A$. baumannii infections among over 5000 hospitalized patients across 250 hospitals with microbiology data based on the Premier HealthCare Database between 2014 and 2019. Outcomes in patients with CR A. baumannii infections were worse than in those with CS infections. Patients 


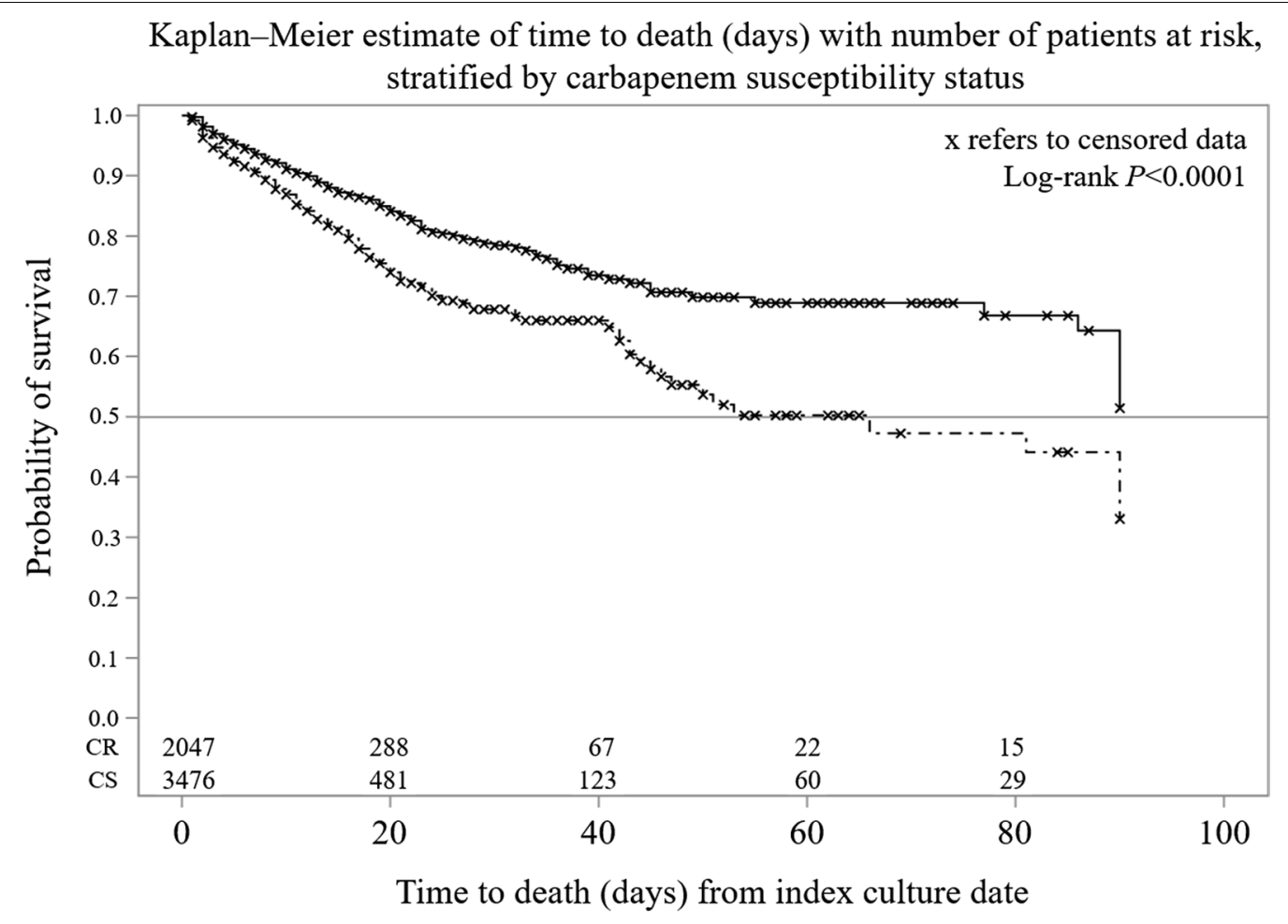

$\mathrm{CS} \quad-\cdot-\cdot-\cdot-\cdot-\cdot \mathrm{CR}$

Fig. 2 Kaplan-Meier analysis of survival probability in patients with Acinetobacter baumannii infections. CR carbapenem resistant, CS carbapenem susceptible

with CR infections had an increased in-hospital mortality, increased length of overall hospital and infectionassociated stay, a lower proportion of survivors being discharged home, and more frequent readmissions with isolation of the same pathogen. Increased mortality with $\mathrm{CR}$ infections persisted even after adjusting for other factors. Interestingly, despite more frequent ICU admission during index hospitalization, the length of ICU stay and ICU-related hospital charges were similar between CR and CS A. baumannii infections.

Overall, the absolute difference in in-hospital mortality rates between CR and CS infections was approximately 6\% (CR 16\% vs CS 10\%). However, the Kaplan-Meier analysis suggested that long-term probability of survival among patients with CR infections decreased at a faster rate than among those with CS infections. We also found that the 30-day and 60-day mortality rates were also increased for patients with CR A. baumannii infections (CR $32.2 \%$ vs CS $21.6 \%$ at Day 30, and CR $49.8 \%$ vs CS $31.2 \%$ at Day 60 ). Several studies have shown a higher risk of mortality among patients with CR A. baumannii infections, particularly for infections occurring in the bloodstream and respiratory tract [13], with rates approaching $\sim 50-60 \%$ globally. In the current study, the highest mortality rate in CR A. baumannii was found in bloodstream infections ( $>40 \%)$, followed by respiratory tract infections (22\%).

The most frequent infection sites among CR infections were the respiratory tract $(>40 \%)$ and wounds $(>30 \%)$. Most patients in this study had at least one comorbidity. The most frequently observed conditions were diabetes, chronic respiratory disease, renal disease, and congestive heart failure. Approximately $40 \%$ of CR infections and $20 \%$ of CS infections were admitted from a healthcare facility/origin. While the majority of infections were acquired in the first $48 \mathrm{~h}$ of hospitalization in both groups (58.6\% for CR vs $69.8 \%$ for CS), there was a clear trend of later onset for $\mathrm{CR}$ isolates $(P<0.01)$, suggesting that $\mathrm{CR}$ infections were more likely than CS infections to be nosocomial in origin. Despite improved prevention efforts in US healthcare settings and hospitals, a high rate of hospital-acquired CR A. baumannii infections has also recently been shown in a large patient cohort [17].

A large proportion of patients with CR infections had a stay in ICU and had their index culture taken during ICU stay more frequently than those with CS infections. This suggests a high risk of acquiring CR A. baumannii during an ICU stay, which is consistent with previous 


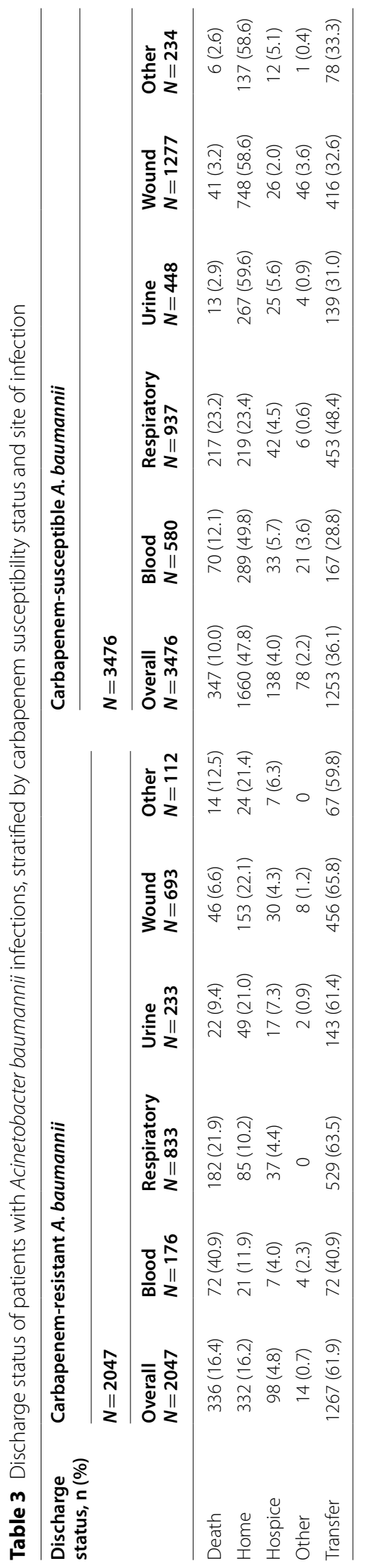


Table 4 Multivariate logistic regression analysis for in-hospital mortality with unadjusted and adjusted odds ratios and 95\% confidence intervals

\begin{tabular}{|c|c|c|c|c|}
\hline Risk factor & Unadjusted OR (95\% Cl) & $P$ value & Adjusted OR $(95 \% \mathrm{Cl})$ & $P$ value \\
\hline \multicolumn{5}{|l|}{ Age group (reference group age 18-35) } \\
\hline $36-55$ & $1.73(1.09 ; 2.73)$ & 0.02 & $1.67(1.03 ; 2.71)$ & 0.04 \\
\hline $56-75$ & $3.33(2.16 ; 5.14)$ & $<0.01$ & $2.45(1.54 ; 3.91)$ & $<0.01$ \\
\hline$>75$ & $4.33(2.76 ; 6.79)$ & $<0.01$ & $3.53(2.17 ; 5.74)$ & $<0.01$ \\
\hline \multicolumn{5}{|l|}{ Sex } \\
\hline Male vs female & $0.87(0.74 ; 1.02)$ & 0.09 & $0.94(0.79 ; 1.13)$ & 0.51 \\
\hline \multicolumn{5}{|l|}{ Race (reference group "black") } \\
\hline White & $1.15(0.94 ; 1.42)$ & 0.18 & $1.14(0.91 ; 1.43)$ & 0.27 \\
\hline Other & $1.47(1.11 ; 1.96)$ & 0.01 & $1.57(1.15 ; 2.15)$ & $<0.01$ \\
\hline Unknown & $0.79(0.36 ; 1.76)$ & 0.57 & $0.56(0.24 ; 1.31)$ & 0.18 \\
\hline \multicolumn{5}{|l|}{ Infection site (reference group "Urine") } \\
\hline Blood & $4.27(2.90 ; 6.28)$ & $<0.01$ & $3.82(2.56 ; 5.71)$ & $<0.01$ \\
\hline Respiratory & $5.37(3.76 ; 7.68)$ & $<0.01$ & $2.66(1.80 ; 3.92)$ & $<0.01$ \\
\hline Wound & $0.85(0.57 ; 1.28)$ & 0.44 & $0.89(0.58 ; 1.34)$ & 0.57 \\
\hline Other & $1.13(0.64 ; 1.99)$ & 0.67 & $0.99(0.55 ; 1.78)$ & 0.98 \\
\hline \multicolumn{5}{|c|}{ Days between admission and index culture (prior to admission) } \\
\hline Same day as admission & $1.29(0.76 ; 2.19)$ & 0.34 & $0.88(0.5 ; 1.55)$ & 0.66 \\
\hline 2 days & $1.27(0.73 ; 2.21)$ & 0.41 & $0.78(0.42 ; 1.43)$ & 0.42 \\
\hline 3 days & $1.75(0.96 ; 3.19)$ & 0.07 & $0.96(0.50 ; 1.86)$ & 0.92 \\
\hline 4 days & $2.20(1.16 ; 4.16)$ & 0.02 & $1.23(0.61 ; 2.47)$ & 0.56 \\
\hline 5 days & $2.45(1.28 ; 4.67)$ & 0.01 & $1.12(0.55 ; 2.27)$ & 0.76 \\
\hline$\geq 6$ days & $3.41(2.01 ; 5.79)$ & $<0.01$ & $1.57(0.87 ; 2.81)$ & 0.13 \\
\hline \multicolumn{5}{|l|}{$\mathrm{CCl}$ group (reference group $\mathrm{CCl}=0$ ) } \\
\hline 1 & $1.52(0.99 ; 2.33)$ & 0.06 & $1.10(0.70 ; 1.72)$ & 0.69 \\
\hline 2 & $1.82(1.22 ; 2.72)$ & $<0.01$ & $1.37(0.90 ; 2.11)$ & 0.14 \\
\hline $3-5$ & $2.54(1.77 ; 3.63)$ & $<0.01$ & $1.72(1.17 ; 2.54)$ & 0.01 \\
\hline$>5$ & $4.31(2.98 ; 6.21)$ & $<0.01$ & $2.73 ;(1.83 ; 4.08)$ & $<0.01$ \\
\hline \multicolumn{5}{|l|}{ Admission source (reference group "Home") } \\
\hline Nursing & $1.72(1.29 ; 2.31)$ & $<0.01$ & $1.22(0.88 ; 1.68)$ & 0.23 \\
\hline Transferred from other facilities & $1.39(1.15 ; 1.68)$ & $<0.01$ & $1.17(0.95 ; 1.45)$ & 0.14 \\
\hline Other & $0.48(0.21 ; 1.09)$ & 0.08 & $0.63(0.26 ; 1.51)$ & 0.30 \\
\hline \multicolumn{5}{|l|}{ Infection associated ICU utilization } \\
\hline Yes vs no & $4.77(4.01 ; 5.68)$ & $<0.01$ & $2.93(2.40 ; 3.57)$ & $<0.01$ \\
\hline \multicolumn{5}{|l|}{ Carbapenem susceptibility status } \\
\hline CR vs CS & $1.77(1.51 ; 2.08)$ & $<0.01$ & $1.42(1.15 ; 1.75)$ & $<0.01$ \\
\hline \multicolumn{5}{|c|}{ Patient received any active drug within $72 \mathrm{~h}$ of index culture date } \\
\hline No active antibiotic ${ }^{a}$ vs active antibiotic ${ }^{b}$ & $1.16(0.98 ; 1.36)$ & 0.08 & $1.03(0.84 ; 1.27)$ & 0.77 \\
\hline
\end{tabular}

CCI Charlson Comorbidity Index, CI Confidence interval, CR Carbapenem resistant, CS Carbapenem susceptible, ICU intensive care unit, OR odds ratio

a The antibiotic is considered as "active" if Acinetobacter baumannii was "susceptible" based on susceptibility testing result. The category included tigecycline and colistin without available susceptibility testing result

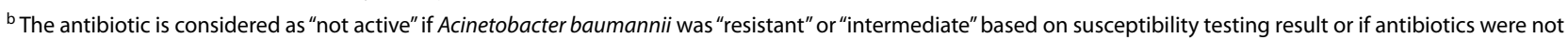
tested for susceptibility or the missing testing results cannot be imputed based on the algorithm in Additional file 7: Table S6

reports [7]. This is an important finding as there was increased mortality when patients had an ICU stay during index hospitalization, which was observed among both CR and CS infections. A previous US study assessing patients in the ICU with bloodstream infections due to Acinetobacter spp. had suggested that inappropriate antibiotic use, particularly for CR Acinetobacter spp., was strongly associated with increased mortality risk [18]. Interestingly, in this analysis, no association between receipt of in vitro active antibiotics within 
the first $72 \mathrm{~h}$ after culture collection and mortality was demonstrated, even though this occurred with four times greater frequency ( $61 \%$ vs $15 \%$ ) than in patients with CS isolates. While this analysis was not intended to address the impact of active therapy on outcomes, potential reasons for this unexpected finding include the inclusion of patients presenting with a wide degree of initial severity of illness, the large proportion of patients with less severe infection types (i.e., wound and urine), or the inferior definitive therapy options available for the treatment of carbapenem-resistant $A$. baumannii, given the extensively drug-resistant nature of most isolates.

Compared with CS patients, CR patients incurred significantly higher charges for overall LOS and infection-associated LOS, but similar charges for ICU-LOS and infection associated ICU-LOS. This may indicate that infections increased the overall hospital burden. The increased LOS and infection-associated LOS may explain the higher amount of charges for CR infections in general. Because ICU-associated LOS was not significantly different between CR and CS infections, it may be the explanation for similar charges for ICU stay by hospitals or higher mortality in CR patients resulted in a short ICU-LOS.

Readmission among patients surviving the index hospitalization may be linked to infectious or noninfectious causes because comorbidities were common in all patients. The overall readmission rates were similar between CR and CS infections. However, CR patients were more likely to be readmitted and have isolation of a Gram-negative organism, or with A. baumannii specifically, which may suggest suboptimal antibiotic treatment during the index hospitalization and/or persistence of pathogens. Further investigation into optimal treatment strategies for $A$. baumannii are clearly warranted.

One limitation of the current analysis is that specific antibiotic regimens were not evaluated and therefore an association between different regimens and patient outcomes was not assessed. Further analyses are required to determine which antibiotic regimens are optimal for the treatment of A. baumannii infections and whether agent selection can positively impact mortality, LOS, and/or readmission rates. An additional limitation is that infections were defined in this analysis by the type of culture sample and the administration of antibiotics at the time of that index culture. While the prescription of antibiotics during the 48-72-h window surrounding the index culture is highly suggestive of infection, whether there was an infection at the culture site and/or whether $A$. baumannii was considered the primary pathogen cannot be ascertained from such an analysis.

\section{Conclusions}

Patients with CR A. baumannii infections had an increased disease burden and higher rate of nosocomial infections, increased LOS and infection-associated LOS, more frequent ICU admission, and higher readmission rates than patients with CS infections. Mortality was also significantly higher for CR A. baumannii infections, particularly among those with bloodstream infections.

\section{Abbreviations \\ CCI: Charlson comorbidity index; CDC: Centers for Disease Control and Preven- tion; Cl: Confidence interval; CR: Carbapenem resistant; CS: Carbapenem susceptible; ICU: Intensive care unit; LOS: Length of stay; MDR: Multidrug resistant; OR: Odds ratio; SD: Standard deviation; SNF: Skilled nursing facility; US: United States.}

\section{Supplementary Information}

The online version contains supplementary material available at https://doi. org/10.1186/s12879-021-07024-4.

Additional file 1: Fig. S1. Distribution of site of carbapenem resistant (CR) or carbapenem susceptible (CS) Acinetobacter baumannii infections in hospitalized patients.

Additional file 2: Table S1. Charges associated with length of stay and intensive care unit utilization

Additional file 3: Table S2. Discharge status of patients with Acinetobacter baumannii infections, stratified by carbapenem susceptibility status, infection site, and intensive care unit stay.

Additional file 4: Table S3. Univariate analysis for patient characteristics, infection-associated ICU utilization, carbapenem susceptibility status, and administration of active or inactive antibiotics for in-hospital mortality.

Additional file 5: Table S4. Readmission rates of patients with Acinetobacter baumannii infections for survivors of index hospitalization, stratified by carbapenem susceptibility status, discharge location, and infectious agent.

Additional file 6: Table S5. Readmission rates of patients with Acinetobacter baumannii infections for survivors of index hospitalization, stratified by carbapenem susceptibility status, site of infection, and infectious agent.

Additional file 7: Table S6. Algorithm to impute antibiotic susceptibility for Acinetobacter baumannii without susceptibility testing result for treatment given within $72 \mathrm{~h}$ of index culture.

\section{Acknowledgements}

Editorial support was provided by Highfield, Oxford, United Kingdom, sponsored by Shionogi Inc., Florham Park, NJ, USA.

\section{Authors' contributions}

Conceptualization of the study: JMP, BC. Data collection, statistical analysis: YZ, HK, BC. Interpretation of the data: JMP, YZ, HK, BC. Writing, approval, and agreement to submit the manuscript: JMP, YZ, HK, BC. All authors read and approved the final manuscript.

\section{Funding}

The work was supported by Shionogi Inc., Florham Park, NJ, USA. The funding body participated in the design of the study, statistical analysis plan, data analysis, and interpretation of the data. No funding to the authors was provided for the preparation of the manuscript.

Availability of data and materials

This study is conducted using an anonymous, commercially available secondary healthcare database called Premier Healthcare Database that meets the US 
HIPPA requirement. The data is not sharable per our license agreement with the data owner.

\section{Declarations}

Ethics approval and consent to participate

Not applicable.

\section{Consent for publication}

Not applicable.

\section{Competing interests}

JMP has served as a consultant for Shionogi, Merck, and Qpex. HK and YZ have received research funding from Shionogi Inc. BC is an employee of Shionogi Inc.

\section{Author details}

${ }^{1}$ College of Pharmacy, University of Michigan, Ann Arbor, MI, USA. ${ }^{2}$ Genesis Research Inc., Hoboken, NJ, USA. ${ }^{3}$ Shionogi Inc., 300 Campus Drive, Suite 100, Florham Park, NJ 07932, USA.

Received: 28 July 2021 Accepted: 30 December 2021

Published online: 06 January 2022

\section{References}

1. Bulens SN, Yi SH, Walters MS, Jacob JT, Bower C, Reno J, et al. Carbapenem-nonsusceptible Acinetobacter baumannii, 8 US metropolitan areas, 2012-2015. Emerg Infect Dis. 2018;24:727-34.

2. Doi Y, Murray GL, Peleg AY. Acinetobacter baumannii: evolution of antimicrobial resistance-treatment options. Semin Respir Crit Care Med. 2015;36:85-98.

3. Asif M, Alvi IA, Rehman SU. Insight into Acinetobacter baumannii: pathogenesis, global resistance, mechanisms of resistance, treatment options, and alternative modalities. Infect Drug Resist. 2018;11:1249-60.

4. Sunenshine RH, Wright MO, Maragakis LL, Harris AD, Song X, Hebden J, et al. Multidrug-resistant Acinetobacter infection mortality rate and length of hospitalization. Emerg Infect Dis. 2007;13:97-103.

5. Ayobami O, Willrich N, Suwono B, Eckmanns T, Markwart R. The epidemiology of carbapenem-non-susceptible Acinetobacter species in Europe: analysis of EARS-Net data from 2013 to 2017. Antimicrob Resist Infect Control. 2020;9:89.

6. Gales AC, Seifert H, Gur D, Castanheira M, Jones RN, Sader HS. Antimicrobial susceptibility of Acinetobacter calcoaceticus-Acinetobacter baumannii complex and Stenotrophomonas maltophilia clinical isolates: results from the SENTRY antimicrobial surveillance program (1997-2016). Open Forum Infect Dis. 2019;6(Suppl 1):S34-46.

7. McCann E, Srinivasan A, DeRyke CA, Ye G, DePestel DD, Murray J, et al. Carbapenem-nonsusceptible Gram-negative pathogens in ICU and nonICU settings in US hospitals in 2017: a multicenter study. Open Forum Infect Dis. 2018;5: ofy241.

8. Centers for Disease Control and Prevention. Antibiotic resistance threats in the United States, 2019. Atlanta, GA: U.S. Department of Health and Human Services, CDC; 2019.

9. Blanco N, Harris AD, Rock C, Johnson JK, Pineles L, Bonomo RA, et al. Risk factors and outcomes associated with multidrug-resistant Acinetobacter baumannii upon intensive care unit admission. Antimicrob Agents Chemother. 2017;62:e01631-e1717.

10. Cai B, Echols R, Magee G, Ferreira JCA, Morgan G, Ariyasu M, et al. Prevalence of carbapenem-resistant Gram-negative infections in the United States predominated by Acinetobacter baumannii and Pseudomonas aeruginosa. Open Forum Infect Dis. 2017;4: ofx176.

11. Cornejo-Juárez P, Cevallos MA, Castro-Jaimes S, Castillo-Ramirez S, Velazquez-Acosta C, Martínez-Oliva D, et al. High mortality in an outbreak of multidrug resistant Acinetobacter baumannii infection introduced to an oncological hospital by a patient transferred from a general hospital. PLOS ONE. 2020;15: e0234684.

12. Tal-Jasper R, Katz DE, Amrami N, Ravid D, Avivi D, Zaldenstein R, et al. Clinical and epidemiological significance of carbapenem resistance in Acinetobacter baumannii infections. Antimicrob Agents Chemother. 2016;60:3127-31.

13. Du X, Xu X, Yao J, Deng K, Chen S, Shen Z, et al. Predictors of mortality in patients infected with carbapenem-resistant Acinetobacter baumannii: a systematic review and meta-analysis. Am J Infect Control. 2019:47:1140-5.

14. Fitzpatrick MA, Suda KJ, Poggensee L, Vivo A, Wirth M, Wilson G, et al. Epidemiology and clinical outcomes associated with extensively drugresistant (XDR) Acinetobacter in US Veterans' Affairs (VA) medical centers. Infect Control Hosp Epidemiol. 2021;42:305-10.

15. Clinical and Laboratory Standards Institute. M100, Performance standards for antimicrobial susceptibility testing, 30th edition. Wayne, PA, USA: CLSI; 2020.

16. US Department of Health and Human Services Office for Human Research Protections. Human Subject Regulations Decision Charts. https://www.hhs.gov/ohrp/regulations-and-policy/decision-charts-2018/ index.html\#c2. Accessed 11 Feb 2020.

17. Jernigan JA, Hatfield KM, Wolford H, Nelson RE, Olubajo B, Reddy SC, et al. Multidrug-resistant bacterial infections in US hospitalized patients, 2012-2017. N Engl J Med. 2020;382:1309-19.

18. Shorr AF, Zilberberg MD, Micek ST, Kollef MH. Predictors of hospital mortality among septic ICU patients with Acinetobacter spp. bacteremia: a cohort study. BMC Infect Dis. 2014;14:572.

\section{Publisher's Note}

Springer Nature remains neutral with regard to jurisdictional claims in published maps and institutional affiliations.

Ready to submit your research? Choose BMC and benefit from

- fast, convenient online submission

- thorough peer review by experienced researchers in your field

- rapid publication on acceptance

- support for research data, including large and complex data types

- gold Open Access which fosters wider collaboration and increased citations

- maximum visibility for your research: over 100M website views per year

At BMC, research is always in progress.

Learn more biomedcentral.com/submissions 